\title{
Spatial Transformations in the Parietal Cortex Using Basis Functions
}

\author{
Alexandre Pouget \\ Institute for Cognitive and Computational Sciences \\ Georgetown University, Washington, DC
}

\section{Terrence J. Sejnowski}

Howard Hughes Medical Institute

The Salk Institute for Biological Studies

University of California, San Diego

\begin{abstract}
Sensorimotor transformations are nonlinear mappings of sensory inputs to motor responses. We explore here the possibility that the responses of single neurons in the parietal cortex serve as basis functions for these transformations. Basis function decomposition is a general method for approximating nonlinear functions that is computationally efficient and well suited for adaptive modification. In particular, the responses of single parietal neurons can be approximated by the product of a Gaussian function of retinal location and a sigmoid function of eye position, called a gain field. A large set of such functions forms a basis set that can be used to perform an arbitrary motor response through a direct projection. We com-
\end{abstract}

\section{INTRODUCTION}

The parietal cortex is thought to contribute to sensorimotor transformations. Located at the crossroads of four sensory systems-visual, auditory, vestibular, and somatosensory-it projects to several frontal and premotor areas (Felleman \& Van Essen, 1991; Andersen et al., 1990a; Blatt, Andersen, \& Stoner, 1990). In humans, lesions of the parietal cortex often result in hemineglect, a syndrome characterized by reduced exploration of the hemispace contralateral to the site of the lesion. Patients with hemineglect have difficulties initiating eye or arm movements toward visual, auditory, or somatosensory stimuli (Heilman, Watson, \& Valenstein, 1985). This deficit is particularly clear in line-cancellation tests, in which the subject is asked to cross out short line segments uniformly spread over a page. Although this task is easy for normal subjects, parietal patients typically fail to cross the lines on the side of the page contralateral to the lesion.

In reaching for an object, or directing gaze toward a visual target, the brain must transform the sensory coordinates of the stimulus into motor coordinates, a point pare this hypothesis with other approaches that are commonly used to model population codes, such as computational maps and vectorial representations. Neither of these alternatives can fully account for the responses of parietal neurons, and they are computationally less efficient for nonlinear transformations. Basis functions also have the advantage of not depending on any coordinate system or reference frame. As a consequence, the position of an object can be represented in multiple reference frames simultaneously, a property consistent with the behavior of hemineglect patients with lesions in the parietal cortex.

illustrated in Figure 1. In the visual cortex, the position of the target is specified in eye-centered, or retinotopic, coordinates. The motor command, on the other hand, is in joint coordinates: the set of joint angles that would bring the hand to the corresponding spatial location.

How does the brain perform such sensorimotor transformations? One possibility is that the task is decomposed in a series of subtransformations in which the position of the target is remapped in various intermediate frames of reference, such as head-centered and bodycentered coordinates (see Fig. 1). This strategy predicts that the cortex should contain multiple representations of the target position in these intermediate frames of reference, each of them involving different neuronal populations. The influential model of spatial transformations in parietal cortex by Zipser and Andersen (1988), and subsequent studies by the same group (Goodman \& Andersen, 1990; Mazzoni \& Andersen, 1995; Andersen, 1995), were based on this assumption.

Although it may be convenient to decompose a transformation into a series of intermediate remappings, as shown in Figure 1, this is not necessarily the most efficient solution nor the only way that biological sys- 


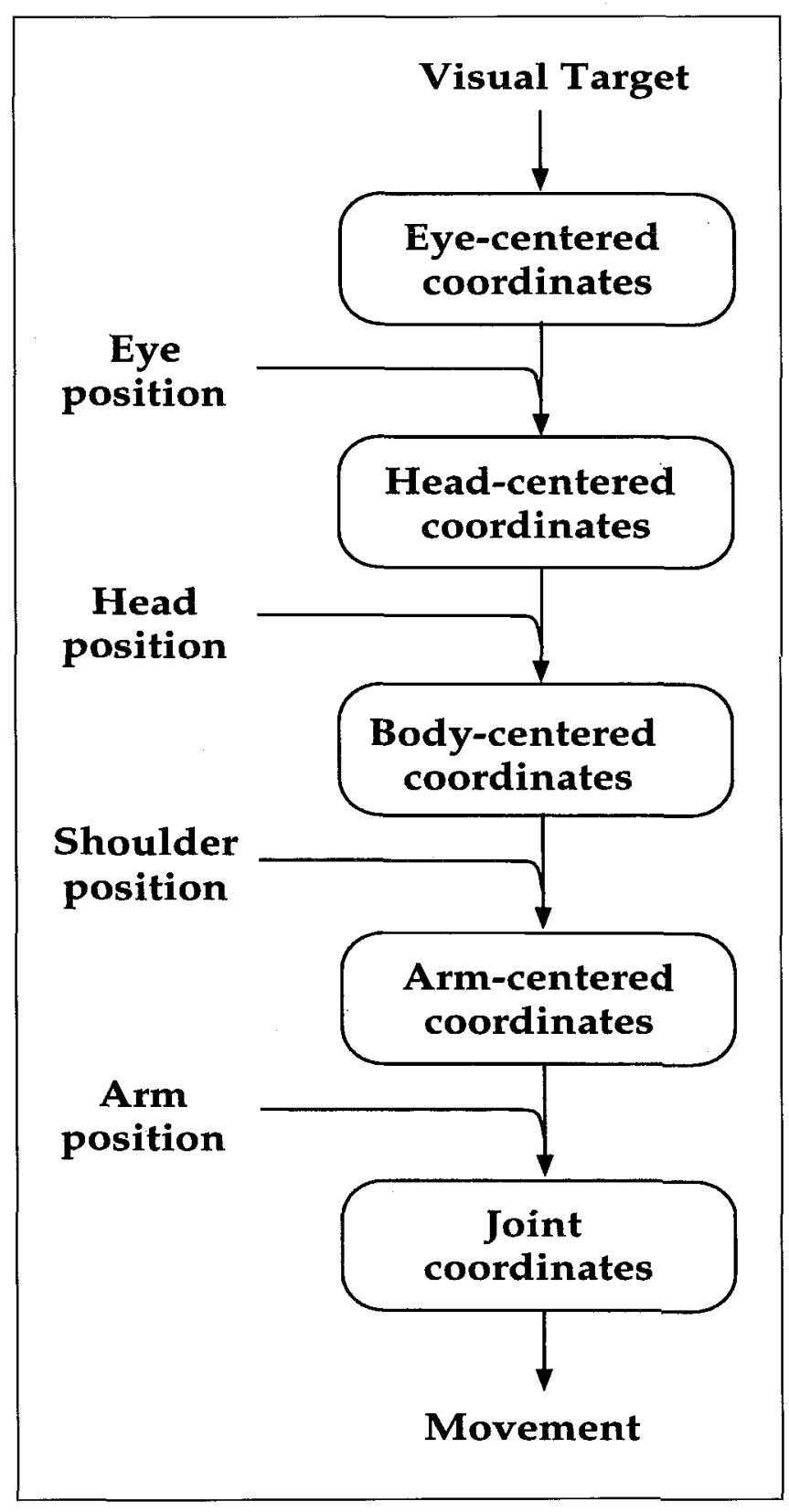

Figure 1. Coordinate transform required to specify an arm movement toward a visual target. The position of the target on the retina is specified in retinotopic coordinates. This position needs to be remapped in joint coordinates in order to move the arm to the corresponding spatial location. This transformation can be decomposed in a series of subtransformations in which the target position is recoded in various intermediate frames of reference.

tems can be organized. We propose, instead, an alternative approach in which sensory inputs, including visual or auditory inputs as well as eye, head, and arm position signals, are encoded in a format suitable for generating motor commands. Our representation is based on the theory of approximation of nonlinear functions (Girosi, Jones, \& Poggio, 1995). We show that the response of parietal neurons is consistent with this hypothesis. The resulting representation, called a basis function repre- sentation, does not encode the location of objects in one particular frame of reference. Instead, the stimulus is represented in multiple frames of reference simultaneously by the same neuronal pool, a feature that could explain many aspects of hemineglect.

In the first part of this paper, we show that sensorimotor transformations are typically nonlinear, a point that we illustrate with a few typical examples. In the second part, we propose that parietal neurons contribute to these transformations by computing basis functions of their sensory inputs. We show that the responses of parietal neurons are consistent with this hypothesis and we describe the results of a simulation in which we demonstrate how the same group of basis function neurons can represent several frames of reference simultaneously. Finally, in the last two sections, we show that encoding the location of an object in one frame of reference at a time, as suggested in Figure 1, using a map or a vectorial code, seems neither appropriate for sensorimotor transformations nor consistent with neurophysiological data.

Part of this work has been published in conference proceedings (Pouget \& Sejnowski, 1995).

\section{Sensorimotor Coordination}

The pattern of muscle activity required to move a limb, or the body, to a specific spatial location is a highly nonlinear function of the sensory inputs. Although the cortex is not believed to specify patterns of muscle activation, it often uses nonlinear representations in the intermediate stages, even if the underlying transformations are actually linear.

Consider, for example, the visuo-somatosensory cells found in the premotor cortex, the putamen, and possibly the ventral intraparietal area (VIP) that have visual receptive fields anchored to the skin, in register with the somatosensory receptive field (Fogassi et al., 1992; Colby \& Duhamel, 1993; Graziano \& Gross, 1993; Graziano, Yap, \& Gross, 1994). When the somatosensory receptive field is located on the face, the visual receptive field is in head-centered coordinates and its position in space must be independent of where the eyes are fixating. What type of computation could be involved in generating this receptive field from the retinotopic visual fields found in the early stages of the visual system?

It might seem that this transformation is linear, since calculating the head-centered location of an object, $\vec{A}$, from its retinal location, $\vec{R}$, and the current eye position, $\vec{E}$, requires a simple vector addition (Groh \& Sparks, 1992; Andersen, 1995):

$$
\vec{A}=\vec{R}+\vec{E}
$$

This equation, however, is only linear in one dimension, since in three dimensions, the geometry of rotation of a spherical body is nonlinear (Westheimer, 1957). Even if we consider this linear approximation-which is 
reasonably accurate for angles less than $40^{\circ}$-other problems arise. Thus, visual receptive fields, whether in the visual system or premotor cortex, are typically limited in size and are approximately bell-shaped. Consequently, the brain does not have access to $\vec{R}$ as a list of numbers - the horizontal and vertical components-but to a set of nonlinear functions of $\overrightarrow{\boldsymbol{R}}$. Likewise, a head-centered receptive field is not $\vec{A}$ itself, but a nonlinear function of $\vec{A}$. Generating such head-centered Gaussian receptive fields from retinotopic gaussian receptive fields requires a nonlinear transformation.

Other sensory remapping problems are formally identical to this case. The generation of eye movements toward auditory and somatosensory targets requires the same type of transformation (Jay \& Sparks, 1987; Groh \& Sparks, 1992; Groh \& Sparks, 1996; Pouget et al., 1993).

In addition to the nonlinearities introduced by remapping the visual field with Gaussian-shaped receptive fields, the brain must also deal with a variety of nonlinear estimation problems. For example, inverse kinematics, the transformation from retinotopic to joint coordinates illustrated in Figure 1, and almost all aspects of arm movement control, require nonlinear mappings (Craig, 1955; Burnod et al., 1992). Nonlinear transformations are the rule rather than the exception in the nervous system.

If the parietal cortex is involved in these transformations, the spatial representations must be capable of approximating nonlinear functions, which provides an important computational constraint on the type of representations that can be used. One possibility, explored in the paper, is that parietal neurons compute basis functions of their sensory inputs.

\section{Gain Fields and Basis Functions}

A nonlinear function, such as $e^{x}$, is typically represented in a computer by a Taylor series, a polynomial expansion that is simpler to compute. This is not the only way to approximate a nonlinear function. An alternative method is to express the function as a linear combination of sines and cosines weighted by numbers called Fourier coefficients. Because sines and cosines can be used to approximate a very large ensemble of nonlinear functions, they are called basis functions. There are many other types of basis functions.

Two classes of basis functions, Gaussians and sigmoids, are especially promising candidates for matching physiological data. Their mathematical properties have been extensively studied (Casdagli, 1989; Moody \& Darken, 1989; Poggio \& Girosi, 1990; Hornik, Stinchcombe, \& White, 1989; Baldi, 1991; Girosi, Jones, \& Poggio, 1995), and they have been used to interpret the responses of single cells in the context of object recognition (Poggio, 1990; Poggio \& Edelman, 1990; Logothetis \& Pauls, 1995) and distance approximations (Pouget \& Sejnowski, 1994).

This basis function framework can also be used to interpret the response of gain-modulated neurons in the parietal cortex (see also Poggio, 1990). If a motor command, $M$, is a nonlinear function of its inputs, it might be generated in the brain by a linear combination of basis

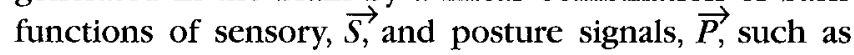
eye, head, and arm positions.

$$
M=\sum_{i} c_{i} B_{i}(\overrightarrow{S,} \vec{P})
$$

where $c_{i}$ are coefficients that depend on the function, $M$, being computed (if the basis functions were sines and cosines, the $c_{i}$ 's would be called Fourier coefficients).

We propose that the responses of parietal neurons behave like the basis functions, $B_{i}(\overrightarrow{S,} \vec{P})$. From a computational perspective, there are several advantages in using basis functions for representing nonlinear transformations in the parietal cortex. First, once the basis functions have been computed, the amount of additional computation needed to obtain a motor command is greatly reduced since any nonlinear function of the input is now only one linear combination away; that is, any nonlinear transformation can be obtained by a single projection. In a sense, the basis functions are closer to the output than a representation that contains separate populations of cells for $\vec{S}$ and $\vec{P}$. Second, the activity of the same neurons can be used to compute several functions, which could be used to drive several motor commands. Third, forming these basis functions during development can be accomplished in a largely unsupervised manner since the choice of basis function is independent of the output functions being computed (Moody \& Darken, 1989).

The responses of single neurons in the posterior parietal cortex are consistent with this hypothesis. These neurons have a visual receptive field whose positions are fixed on the retina, but the amplitudes of the responses to visual stimuli are modulated by eye position (Andersen, Essick, \& Siegel, 1985). Figure 2A shows how the gain of the retinotopic receptive field of a cell changes with eye position. In Figure $2 \mathrm{~B}$, the circles indicate the responses of a single cell to a stimulus flashed in the middle of the receptive field while the monkey fixated nine different locations. The peak response appeared to vary linearly with eye position along a particular direction, left and upward for the cell shown in Figure 2. This is called the gain field, and it corresponds to the receptive ficld of the cell for eye position.

The response of a single cell, like the one shown in Figure 2, can be modeled by the product of a Gaussian function of retinal location with a sigmoid function of eye position. Figure 3 shows the correspondence between the gain field and these idealized response functions. Both Gaussians and sigmoids are basis functions, and it can be shown that the product of two basis functions forms a basis function, but all combinations must be represented (see Appendix for a proof). 
Figure 2. (A) Typical visual receptive field of a parietal neuron shown for two different gaze angles, $e_{x}$. The retinal positions of the receptive fields do not vary with eye position; only the gain of the response changes. (B) Typical gain field of a parietal neuron. The circles show the responses of a single cell to visual stimulation in the center of the receptive field for eye fixations at nine different locations. The diameter of each outer circle is proportional to overall activity, while the inner circle corresponds to visually evoked activity (overall activity minus spontaneous activity). Eye fixation positions

were sampled on a grid with a $20^{\circ}$ spacing, such that the fixation point was straight ahead for the central circle, and $20^{\circ}$ up and $20^{\circ}$ left for the upper left circle. The activity of this cell increased monotonically for eye positions located upward and to the left. This preferred direction is specific to each cell (adapted from Andersen et al., 1985; Andersen \& Zipser, 1988).

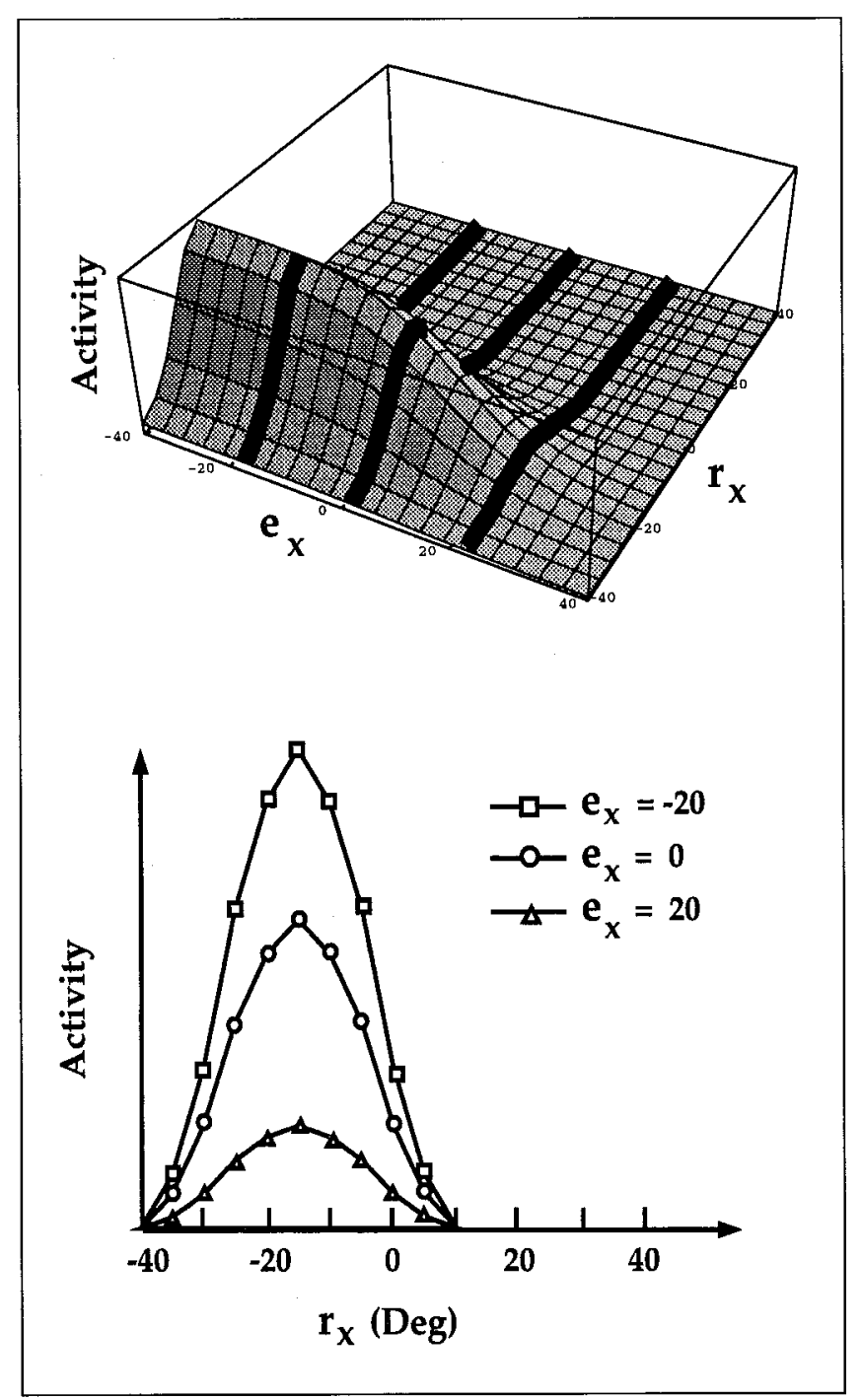

Such cells could be used to generate the skin-centered visual receptive fields of premotor cortex and putamen neurons (Fogassi et al., 1992; Colby \& Duhamel, 1993; Graziano \& Gross, 1993; Graziano, Yap, \& Gross, 1994). Figure 4 shows a 3 -layer network using basis function in the hidden layer to generate units with head-centered receptive fields in the output layer. (Skin-centered is equivalent to head-centered when the receptive field is located on the head.)

The input layer consisted of a one-dimensional retinotopic map similar to that found in the early stages of the visual system, where neurons respond to visual stimuli in a limited region of the visual field. In addition, several input units encode the horizontal position of the eye, $e_{x}$. In the output layer, units were organized in a one-dimensional head-centered map. They responded as a Gaussian function of $a_{x}$, or, equivalently, a Gaussian function of $r_{x}$ $+e_{x}$ [Eq. (1)].

In this one-dimensional case, the activity of all the units in the network can be plotted with respect to the input variables, namely, the retinal position of targets, $r_{x}$, and eye position, $e_{x}$. Examination of the plot for a typical output unit-such as the one shown on top of Figure 4-confirms that the plot is not planar and that a headcentered receptive field is a nonlinear function of the input variables. These nonlinear functions can be generated by a linear combination of the activities of hidden units like the idealized neurons showed in Figure 3.

Figure 3. Response function obtained by multiplying a Gaussian of retinal location with a sigmoid of eye position (top). When sampled at three different gaze angles (thick lines on top graph), the visual receptive field (bottom) shows the same gain modulation as found in the parietal cortex (Figure 2-A). 


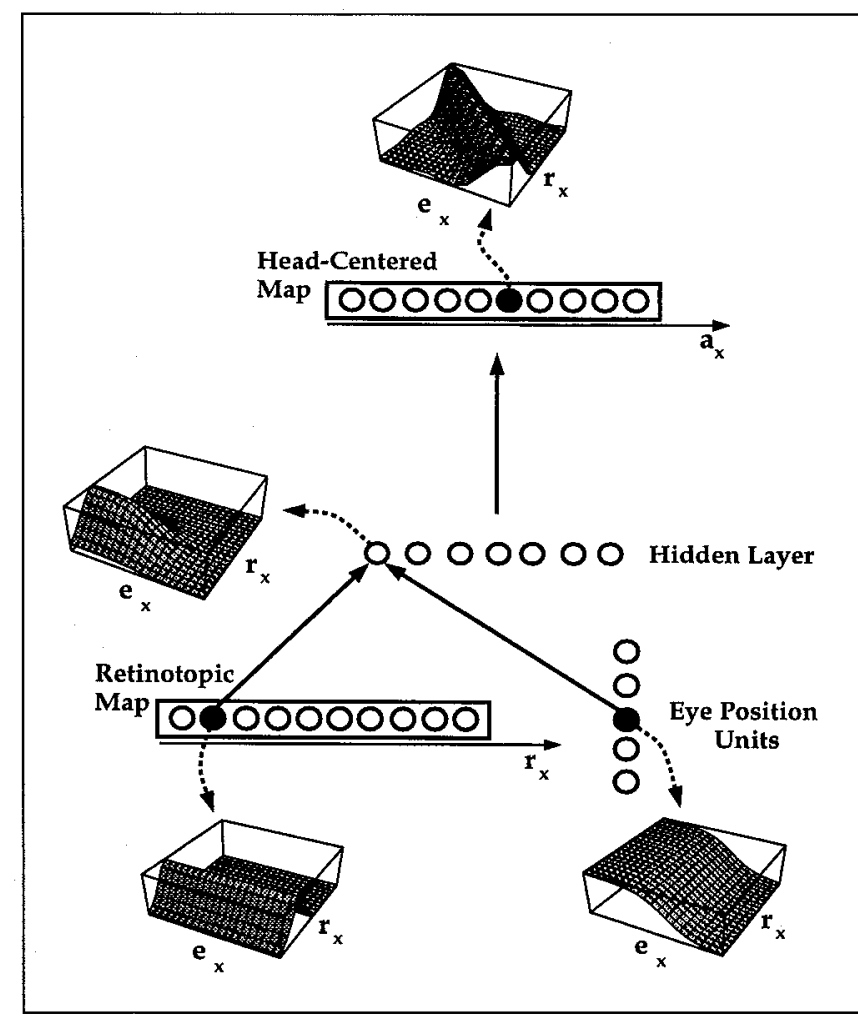

Figure 4. Neural network for transforming a retinotopic map to a head-centered map. The input contains a retinotopic map of the visual input and the output represents a head-centered map. The eye position units have a sigmoidal tuning to eye position and a range of thresholds. The function represented by the network is nonlinear, as illustrated by the fact that the response to $r_{x}$ and $e_{x}$ of the units in the output layer is clearly not a plane. This mapping could be implemented by hidden units that compute the product of a Gaussian of $r_{x}$ with a sigmoid of $e_{x}$. Such units would provide basis functions of the input variables and would respond like gain-modulated neurons found in the parietal cortex.

Note that the same units could be used to generate any other function of $r_{x}$ and $e_{x}$. For example, the parietal cortex is believed to be involved in the control of saccadic eye movements toward visual targets. It projects to two structures, the superior colliculus and frontal eye field, in which many neurons show presaccadic activity, which might be due in part to the parietal input. The motor fields of these neurons are in oculocentric coordinates, which is geometrically equivalent to retinotopic coordinates (Sparks, 1991). A Gaussian retinotopic motor field is another example of a nonlinear function of $r_{x}$ and $e_{x}$-in this particular case, the function depends only on $r_{x}$-and as such it could be generated by a linear combination of parietal neurons' activity.

Therefore, the responses of such basis function cells could be used to control several behaviors simultaneously, such as reaching, and moving the eyes. We demonstrate this point in the next section, in which we generate a Gaussian head-centered receptive field and a Gaussian retinotopic receptive field by a linear combination of the activities of basis function units. The output unit with a retinotopic receptive field could correspond to a presaccadic neuron in the superior colliculus (Sparks, 1991), whereas the one with a head-centered receptive field would be similar to the premotor cortex or VIP neurons with skin-centered receptive field, which are believed to be involved in reaching (Fogassi et al., 1992; Colby \& Duhamel, 1993).

\section{Simulations}

The accuracy with which a sum of basis functions can approximate a transformation depends on the number of basis functions used. Perfect accuracy is only possible in the limit as the number of basis functions becomes infinite, but good approximations can be obtained to many functions with a reasonably small number (Girosi, Jones, \& Poggio, 1995). We illustrate this point by showing how gain-modulated units, similar to the neurons found in the parietal cortex, can be used to generate two output functions: a head-centered and a retinotopic receptive field.

The model used 121 gain-modulated units, corresponding to the hidden units in Figure 4, whose response functions were computed by multiplying Gaussian retinal receptive fields with sigmoid functions of eye position:

$$
\boldsymbol{b}_{i}=\frac{\boldsymbol{e}^{-\frac{\left(r_{x}-r_{x_{i}}\right)^{2}}{2 \sigma^{2}}}}{1+\boldsymbol{e}^{-\frac{e_{x}-e_{x_{i}}}{T}}}
$$

where $b_{i}$ is the activity of unit $i$. The peaks of the Gaussians, $r_{x_{i}}$, were spread uniformly between $-60^{\circ}$ and $60^{\circ}$ in increments of $12^{\circ}$. The standard deviation of the Gaussian, $\sigma$, was fixed at $18^{\circ}$. This corresponds to a radius of $25^{\circ}$, defined as the distance from the peak corresponding to $37 \%$ of maximum activity. An average radius of $22^{\circ}$ has been reported in area 7 a (Andersen, Essick, \& Siegel, 1985). The inflection points of the sigmoids, $e_{x_{i}}$, were also uniformly spread between $-40^{\circ}$ and $40^{\circ}$ in steps of $8^{\circ}$. The slope factor, $T$, was set at $8^{\circ}$. Four typical units used in the simulations are shown at the bottom of Figure 5.

In a second series of simulations, we used a different set of functions, which were obtained by multiplying a Gaussian of $r_{x}$ by a piecewise linear function of $e_{x}$ (similar piecewise linear functions of $e_{x}$ were used in the Zipser and Andersen model, 1988):

$$
\begin{gathered}
\boldsymbol{b}_{i}=e^{-\frac{\left(r_{x}-r_{x_{i}}\right)^{2}}{2 \sigma^{2}}} f_{i}\left(e_{x}\right) \\
f_{i}\left(e_{x}\right)= \begin{cases}\boldsymbol{e}_{x}-e_{x_{t}} & \text { if } e_{x}>e_{x_{t}} \\
0 & \text { otherwise }\end{cases}
\end{gathered}
$$

These functions look similar to the previous ones except that activity does not saturate at a maximum level. The 


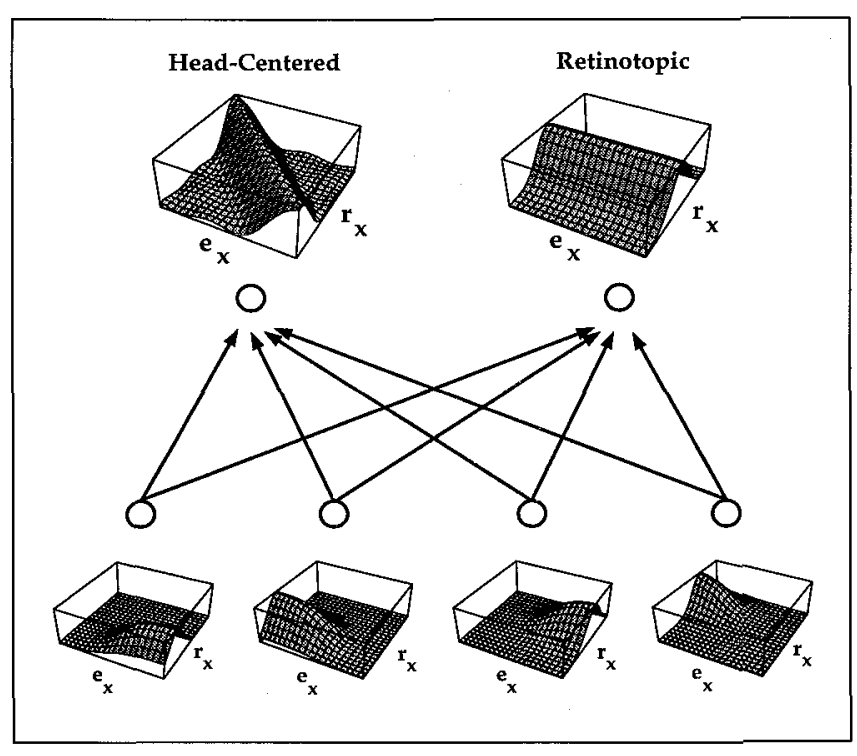

Figure 5. Approximating a head-centered and a retinotopic Gaussian receptive field by the use of the same gain-modulated input units. Only four units are shown, but the approximations were obtained with 121 units.

goal of this second model was to demonstrate that saturation at zero is sufficient as long as the $e_{x_{i}}$ are spread over the range of all possible eye positions.

The two output functions were Gaussian functions of $a_{x}$ and $r_{x}$ respectively, with a standard deviation, $\sigma$, of $18^{\circ}$. The peak in both cases was at $a_{x}=r_{x}=0$. A wide range of peak position could have been approximated equally well by the set of basis functions.

We used a supervised optimization procedure for determining the weights $w_{i}$ between the basis functions and output unit. The optimization procedure, called the delta rule (Widrow \& Hoff, 1960), minimized the square error between our estimation, $o$, and the actual function, $o^{*}$, over all possible examples, $p$ :

$$
E=\sum_{p=1}^{M}\left(o_{p}^{*}-o_{p}\right)^{2}
$$

where

$$
o_{p}=\sum_{i=1}^{121} w_{i} b_{i p}
$$

This procedure was used only to find such a set of weights and not to model the actual process that might be used in the brain to determine these weights, although the rule we used is quite simple and could be easily implemented in neural hardware. Even simpler correlation rules can be used, as demonstrated by Salinas and Abbott (1995).

The training set was composed of 441 pairs of retinal position, $r_{x}$ and eye position, $e_{x}$, selected from 21 different retinal locations within the range $-40^{\circ}$ and $40^{\circ}$, and as many eye positions between $-20^{\circ}$ and $20^{\circ}$. Weights were adjusted until the approximation was, on average, within $3 \%$ of the actual values. Figure 5 shows the resulting approximation for a head-centered and a retinotopic receptive field when using products of Gaussians and sigmoids. Identical results (not shown) were obtained when using the second type of basis functions.

One might have thought that recovering a retinotopic receptive field from the activity of basis function units is trivial since these units already have a Gaussian retinotopic receptive field. However, it is worth noting that the retinotopic receptive field was recovered with the same type of transformation as the one used for the head-centered receptive field, namely a linear transformation. Therefore, the two frames of reference coexist in the basis function representation on equal footing. An infinite number of potential frames of reference are implicit in this representation, and any of them could be extracted with only a single linear projection.

Note that the basis function units contain multiple frames of reference, but the output units extract only the coordinates needed for the behavior they control. Therefore, a given behavior has access to only one frame of reference. In the case of eye movements, this model assumes that the oculomotor coordinates are retinotopic. Consequently, our model cannot deal with double saccade toward remembered targets, a task that would require some form of head-centered coordinates. However, this problem can be easily fixed by using a moving hill mechanism like the one proposed by Droulez and Berthoz (1991), which is known to solve the doublesaccade paradigm.

Finally, the ability of the basis function network to generate any nonlinear function in the output stage is a defining characteristic of this representation. Had the hidden units in the network been linear, a nonlinear function could not have been well approximated by a linear combination of the hidden units.

\section{Response Properties Required by Basis Function Representations}

For mathematical convenience, we used basis functions that were the products of Gaussian with sigmoid or linear-rectified functions. Few neurons in the parietal cortex have response functions that fit perfectly with these functions. These idealized responses are not strictly required, however, but there are at least two necessary conditions that must be met:

1. The selectivities to $\vec{R}$ and $\vec{E}$ should interact nonlinearly.

2. The visual receptive fields as well as the gain fields should be nonlinear functions of $\vec{R}$ and $\vec{E}$.

These conditions are not sufficient, in a mathematical sense, to insure that the functions are basis functions, but a very large number of functions satisfying these requirements do form basis sets (see Hornik et al., 1989; 
and Girosi et al., 1995). We have confirmed this point empirically, through computer simulations, by using a wide variety of such functions.

Two common classes of functions violate one of these conditions: sums of linear or nonlinear functions of $\vec{R}$ and $\vec{E}$, and two independent sets of functions of $\vec{R}$ and functions of $\vec{E}$. It is shown in the Appendix that neither of these two classes of functions forms a basis set, thereby demonstrating that the two conditions above are indeed necessary to obtain a basis set (see Part 2). This is related to the fact that basis functions of several variables must combine these variables in such a way that they are no longer linearly separable.

This result entails that several potentially interesting functions cannot be used for a basis set, including a representation in which separate populations of cells are

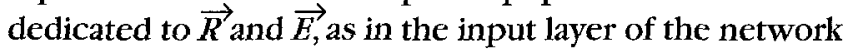
in Figure 4. Nor would a representation using units whose responses were linear in $\vec{R}$ or $\overrightarrow{E,}$ such as Gaussians of $\vec{R}$ multiplied by linear functions of $\vec{E}$ (Andersen, Essick, \& Siegel, 1985).

Another example of function that does not constitute a basis set is the set formed by functions that are the sums of one Gaussian of $\vec{R}$ and one sigmoid of $\vec{E}$. This might appear counterintuitive since this set is similar to the one used in the simulations: the products of Gaussians and sigmoid functions. However, since the set is made up of sums of Gaussians and sigmoids, linear combinations of these functions can only produce a function which is itself a sum of several Gaussians and sigmoids. The resulting function can therefore be decomposed into the sum of one function of $\vec{R}$ plus one function of $\vec{E}$. Most functions of $\vec{R}$ and $\vec{E}$, such as $e^{R+E}$, cannot be decomposed into a sum of two functions.

It is therefore essential to establish that the responses of a large percentage of parietal neurons are consistent with the two criteria above. Without a theory of basis functions, there would be no reason to test for these properties.

\section{Condition 1: Nonlinear Interaction}

Determining the exact form of the interaction between retinal and eye position selectivities for parietal neurons requires a complete mapping of the visual receptive field for several fixation positions. Andersen et al. (1985) have performed this analysis on seven cells only, but for each of these cells, they found that the response is best modeled by a multiplication between selectivities. This is quite clear for the four cells shown in Figure 6. If the cell simply added eye position with the visual input, the entire retinal receptive field should move upward or downward with change in eye position. Instead, the firing rate of the cell is modified most at the peak response, and responses close to zero are barely affected.

Additional evidence can be obtained by examining the

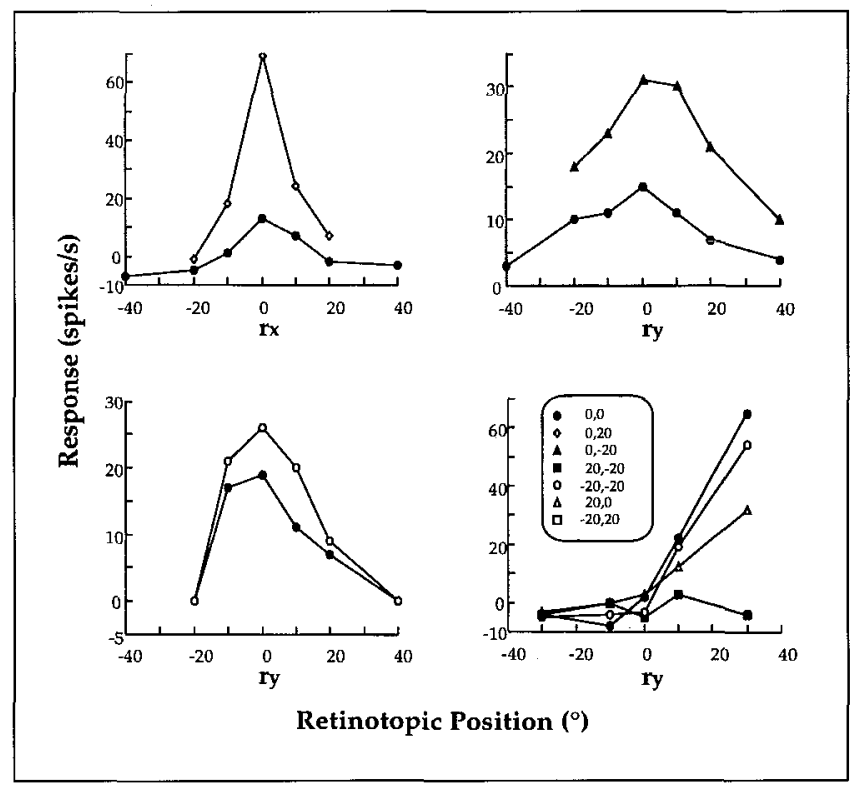

Figure 6. Four typical visual receptive fields of parietal neurons. Each receptive field is shown for several gaze angles (adapted from Andersen et al., 1985).

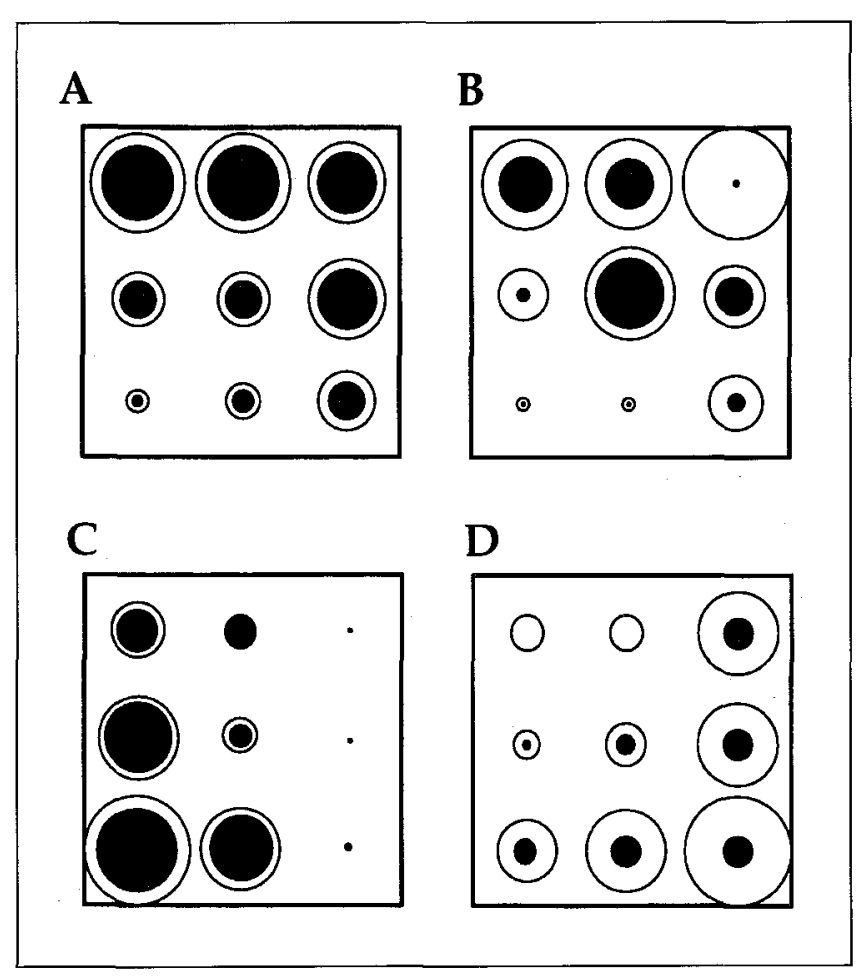

Figure 7. Four typical gain fields of parietal neurons. Notice that gain fields (B) and (C) show clear signs of saturation. See Figure 2B for explanation of circles (adapted from Andersen et al., 1988).

covariation of visually evoked activity (black circle in Fig. 7) and eye position activity (white ring) in the gain fields. Andersen and Zipser (1988) performed this analysis on $78 \%$ of the gain-modulated neurons recorded in area $7 \mathrm{a}$ and the lateral intraparietal area (LIP). They found that of these, $43 \%$ had gain fields consistent with 
a multiplicative interaction, but not with a simple addition (Andersen \& Zipser, 1988, Fig. 13B). For an additional $28 \%$ of the cells, the interaction might be even more complex. Hence, the gain field depicted in Figure $7 \mathrm{~B}$ cannot be explained by a simple multiplicative interaction. The fact that the visually evoked activity decreased as the eyes moved up and to the right indicates that eye position alone is sufficient to saturate the response of the cell at maximum firing rate. Andersen and Zipser (1988) concluded that such responses were consistent with a sigmoid activation function for the gain field. The remaining $29 \%$ of the cells are consistent with both a linear or a nonlinear interaction, and more measurements would be required to decide. We conclude that the responses of at least $43+28=71 \%$ of the parietal neurons tested satisfied the first requirement: the visual and eye position selectivities interacted nonlinearly.

\section{Condition 2: Nonlinear Dependence}

Visual receptive fields of parietal neurons are typically smooth and nonlinear functions of $\vec{R}$. Gaussian functions or sums of Gaussians provide good models of their profile, as seen in Figure 6 . The eye position selectivity, however, which is called the gain field (Figure 2B), appears to be a linear function of $\vec{E}$ which would not satisfy the second condition. Since our model requires nonlinear basis functions, we need to examine more closely the gain fields of parietal neurons to see if there are nonlinearities.

Andersen and Zipser (1988) and Andersen et al. (1990b) performed a linear regression analysis on a large sample of parietal neurons. This analysis revealed that about $40 \%$ of the cells had a planar gain field, another $40 \%$ had a planar component in their gain field (they were not purely planar but were monotonically increasing in one direction of space), and the final $20 \%$ had nonplanar gain fields (Andersen \& Zipser, 1988; Andersen et al., 1990b; see Figure 7 for four examples). Although linear regression analysis revealed that about $80 \%$ of the gain fields were either planar or have a planar component, a closer analysis showed that this percentage does not necessarily entail that most cells are really linear.

Figure 8 illustrates that sampling a sigmoid at nine symmetrical positions results in a gain field that would appear to be planar if tested with a linear regression analysis. If the inflection point of the sigmoid is not exactly at $e_{x}=e_{y}=0$, the resulting gain field would look less linear, but would still be monotonic, and a linear regression analysis would find that there is a statistically significant linear component. Therefore, even though $80 \%$ of the neurons had gain fields that were either planar or had a planar component, this is also consistent with sigmoidal gain fields. The remaining $20 \%$ classified as nonplanar had a peak of activity at one of the nine

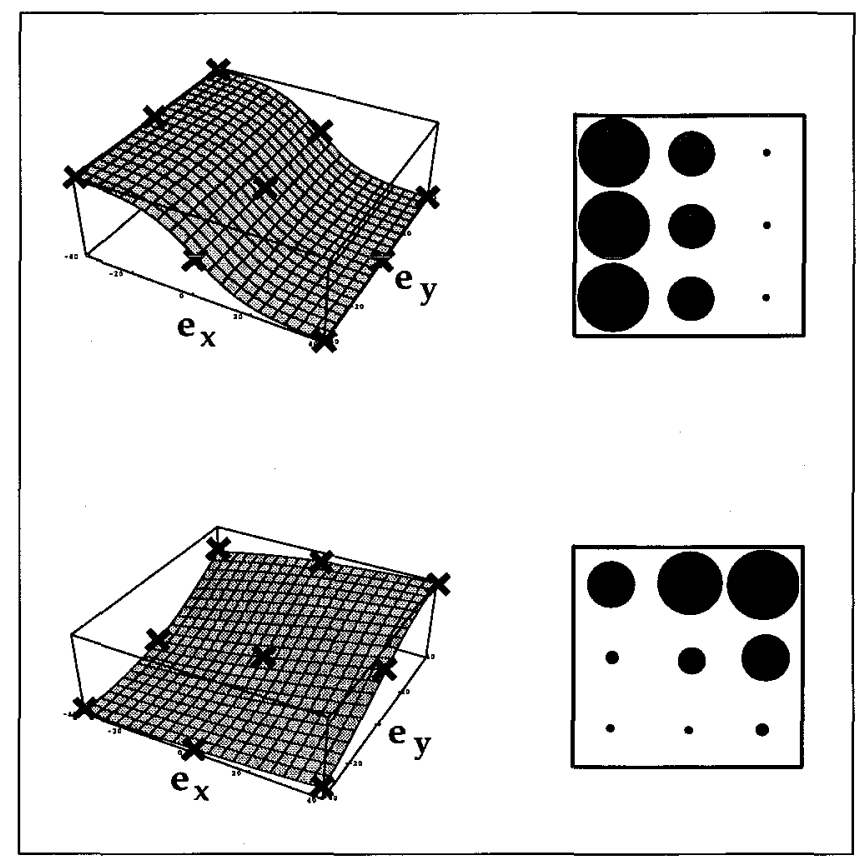

Figure 8. Examples of two sigmoid functions sampled at nine eye positions (crosses) showing that they are nearly planar gain fields.

sampling positions (Andersen \& Zipser, 1988; Andersen et al., 1990b). These might be just as useful as the others and are consistent with the second condition above.

Evidence for saturation of the response of a neuron at a minimum or maximum firing rate within the working range of eye positions or retinal locations could distinguish between genuinely planar tuning and sigmoidal gain fields. Linear tuning (also called a vectorial code, as shown later) requires that saturation should not occur within the physical limits of eye position (around $\pm 50^{\circ}$ ).

Response saturation was tested by examining data from neurons in parietal cortex. Saturation at maximum firing rate is difficult to demonstrate because it requires a large number of measurements. Saturation at zero, on the other hand, can be estimated by linearly extrapolating the gain field of each cell beyond the experimental sample points to determine which eye position would silence the cell. This is only an estimation, however, since we can only surmise that cells keep responding linearly outside of the range of eye position tested.

We performed this analysis on the gain fields of 174 cells recorded in the parietal area $7 \mathrm{a}$ by Andersen, Essick, and Siegel, 1985. An example of one of these gain fields is shown in Figure 2B. Gain fields were fitted with planes such that the activity, $a$, of each cell was approximated with:

$$
a=\alpha e_{x}+\beta e_{y}+\gamma
$$

where $\alpha, \beta$, and $\gamma$ were obtained with a linear regression analysis (see Andersen \& Zipser, 1988 for more details on this procedure). The minimum eye deviation from the 
straight-ahead for which the cell would stop firing is given by:

$$
\theta=\frac{\gamma}{\beta} \cos \left[\operatorname{atan}\left(\frac{\alpha}{\beta}\right)\right]
$$

Figure 9 shows the measured distribution of $\theta$ for neurons in parietal cortex. Although the distribution is not uniform, many cells saturate within $\pm 40^{\circ}$, the working range of normal saccadic eye movements.

The two large peaks on the sides are for cells that intersect beyond $\pm 80^{\circ}$. These cells might be either true linear cells or cells with sigmoidal tuning that are near saturation at maximum firing rate around $\pm 20^{\circ}$. Additional measurements are needed to distinguish between these two possibilities.

The data in Figure 9 provide clear evidence of saturation at zero firing rate within working range of eye positions. It is not yet possible to conclude, though, that the tuning to eye position is sigmoidal since we do not have enough data to demonstrate saturation at maximum firing rate.

A recent study by Squatrito and Maioli (1996) suggests that saturation at the maximum firing rate can occur. They reported that the tuning of pure eye position cells in area $7 a-$ cells responding to eye position only-is best described by sigmoidal functions. Since these cells probably provide the eye position signal to the gainmodulated visual cells in these areas, the resulting gain fields may reflect the tuning properties of these pure eye position cells.

Short of concluding that the gain fields are sigmoidal, we can still conclude that they are nonlinear within the

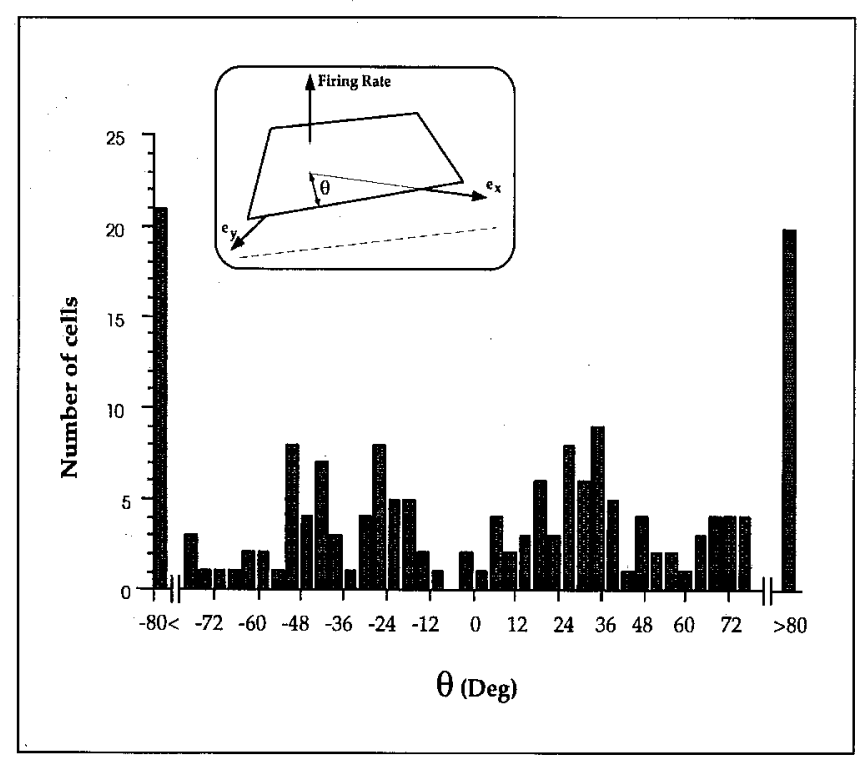

Figure 9. Histogram of the gain field $\theta$, of 174 cells recorded in area 7 a (data from Andersen, Errick, \& Siegel, 1988). As illustrated in the inset, $\theta$ was defined as being the smallest eye deviation from straight ahead that would silence the cell.

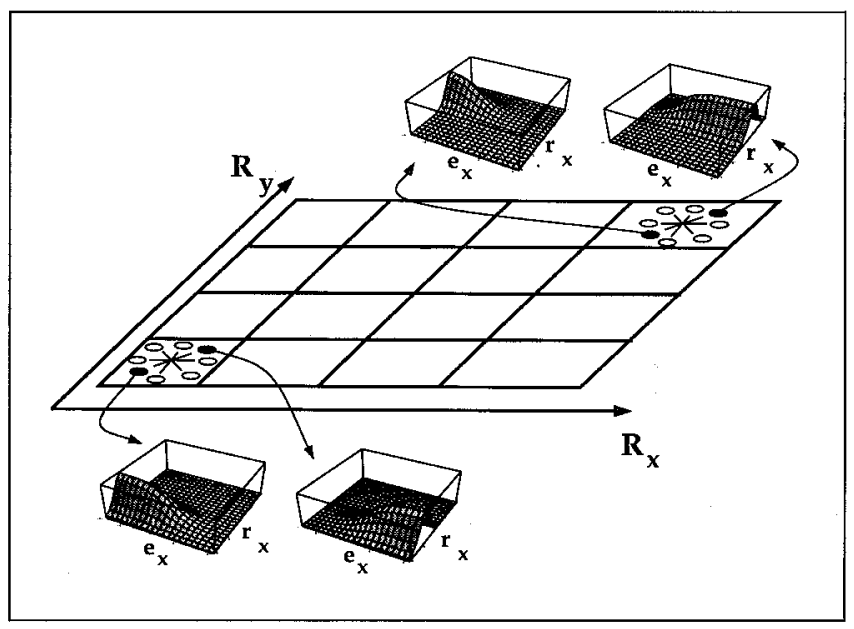

Figure 10. A spatial representation using basis functions. Units have a Gaussian retinal receptive field multiplied by a sigmoid of eye position. For each retinal location, a small population of units represents all possible gaze angles. The spatial location of an object is represented by patterns of activity in this map.

normal range of eye positions. We have demonstrated by simulation that units with piecewise linear gain fields that saturate at zero ("hinge" units) produce good estimates of nonlinear mappings.

The receptive field properties of parietal neurons are therefore broadly consistent with our basis function hypothesis. The schematic view in Figure 10 shows basis function units for all possible combinations of retinal and eye position selectivities; any function of these inputs can be computed as a weighted sum of this basis set.

\section{COMPARISON WITH OTHER REPRESENTATIONS}

If the brain decomposes a coordinate transformation into a series of subtransformations in which the position of an object is remapped in various frames of reference (see Figure 1), then there should exist multiple representations of object position, each of them encoding the location of an object in some frame of reference, such as head-centered coordinates. The location of an object relative to the head is a vector; that is, we can represent the location of an object with respect to an origin fixed on the head. There are many ways to represent a vector. The two most common types of distributed representation for vectors are computational maps (Knudsen, du Lac, \& Esterly, 1987) and vectorial codes (Soechting \& Flanders, 1992; Goodman \& Andersen, 1990; Touretzky, Redish, \& Wan, 1993).

\section{Map Representation}

Several brain structures use two-dimensional maps of neurons to represent vectors, including the retinal posi- 


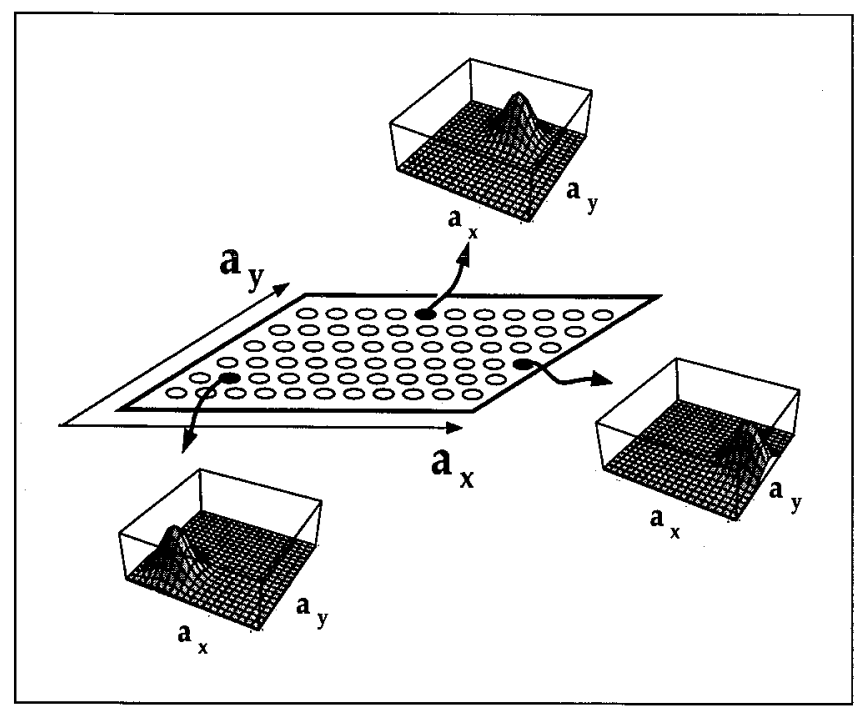

Figure 11. Computational map for the head-centered location of an object, $\vec{A}$. Each unit responds to a limited range of $a_{x}$ and $a_{y}$ with a Gaussian tuning.

tion of visual stimuli in area V1 and the direction and amplitude of the next saccadic eye movement in the superior colliculus (Lee, Rohrer, \& Sparks, 1988). The parietal cortex may, in a similar manner, represent the head-centered location of an object in a two-dimensional map (see Fig. 11). Each neuron in the map would discharge for a limited range of values of $a_{x}$ and $a_{y}$ so that their receptive fields would be fixed in head-centered coordinates. When the head and body are fixed, neurons in such a map would respond to visual stimulation at a particular location in space, regardless of eye position.

There is preliminary evidence that some bimodal neurons in the ventral intraparietal area (VIP) might use such a code (Colby \& Duhamel, 1993). Outside of VIP, however, only a few cells have head-centered receptive fields (Galletti, Battaglini, \& Fattori, 1993) and it is generally believed that this kind of representation is not predominant (Andersen, 1989).

\section{Vectorial Representation}

\section{Definition}

The components of a two-dimensional vector are typically the projections of the vector along the horizontal and vertical axes. The choice of the axes is, however, arbitrary. The same two-dimensional vector can be represented by its projection on any pair of axes as long as they are independent (see Fig. 12). The parietal cortex could encode the head-centered position of object, $\vec{A}$, by projections along vectors, so that the firing rate of a neuron would report the projection of $\vec{A}$ along its preferred direction. Then the activity, $o$, of a neuron can be modeled as:

$$
o=\vec{W}_{a}^{T} \vec{A}=\|\vec{W}\|\|\vec{A}\| \cos (\theta)
$$

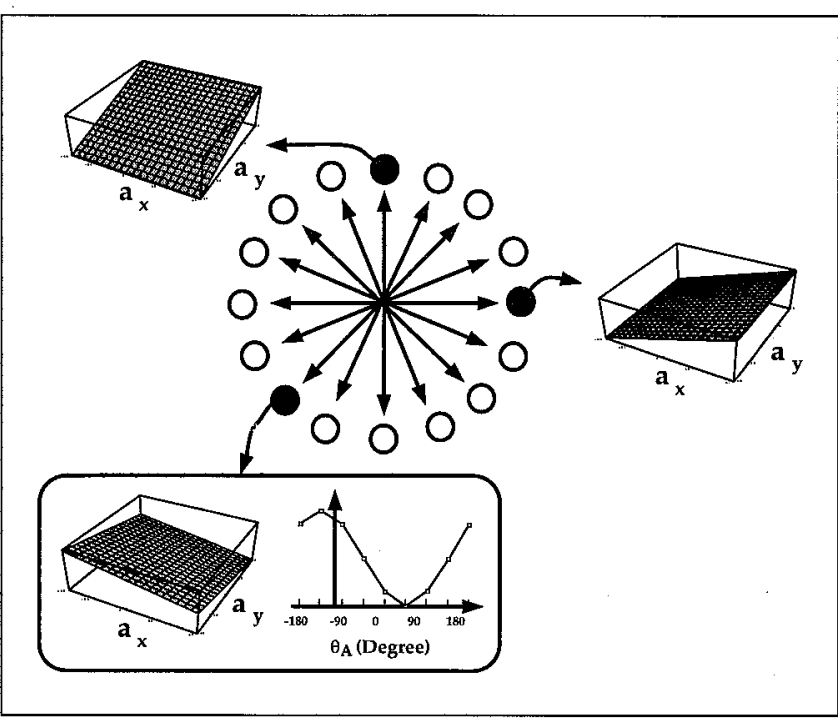

Figure 12. Vectorial representation for the head-centered location of an object, $\vec{A}$. Each neuron computes the projection of $\vec{A}$ along its preferred direction (central arrows). As a consequence, the tuning curve for $a_{x}$ and $a_{y}$ is planar, whereas the tuning curve to $\theta$ (the angle between $\vec{A}$ and the cell's preferred direction) is a sine function.

where, $\theta$ is the angle between the head-centered position of the object $\vec{A}$ and the vector $\vec{W}_{a} \cdot \vec{W}_{a}$ is called the preferred direction of the cells because the activity is maximum whenever $\theta=0$; that is, when $\vec{A}$ points in the same direction as $\vec{W}_{a}$. This representation predicts that neurons should have a cosine tuning to the direction of the head-centered location of object. Hence, if an object is moved in the visual field along a circle centered on the point of fixation, the response of the neuron should follow a cosine tuning function.

Cosine tuning responses have been reported in the motor cortex for the direction of hand movement, suggesting that the motor cortex uses a vectorial code for the direction of hand movement in extrapersonal space (Georgopoulos et al., 1989; but see Sanger, 1994). The same scheme has been also used by Goodman and Andersen (1990), and Touretzky et al. (1993) to model the encoding of head-centered position of objects in the parietal cortex. Touretzky et al. (1993) called their representation a sinusoidal array to refer to the cosine tuning of the units.

Neurons in the parietal cortex do not receive directly the head-centered position of objects. Instead, they receive signals related to the retinotopic position of object, $\vec{R}$ and the current eye position, $\vec{E}$. Upon substituting $\vec{A}$ from Eq. (1) in Eq. (10), the response of the unit to $\vec{R}$ and $\vec{E}$ is:

$$
o=\vec{W}_{a}^{I}(\vec{R}+\vec{E})=\vec{W}_{a}^{I} \vec{R}+\vec{W}_{a}^{I} \vec{E}
$$

This equation is linear in $\vec{R}$ and $\vec{E}$, which leads to three important requirements regarding the tuning of parietal neurons to the retinal location of the object and the eye position. 


\section{Response Properties Required by Vectorial} Representations

1. The visual and eye position receptive fields of parietal neurons should be planar.

2. The selectivities to $\vec{R}$ and $\vec{E}$ should interact linearly.

3 . The preferred direction for retinal location and eye position should be identical.

The first requirement suggests that the visual receptive field of a neuron should cover the entire visual field and the response to a stimulus should linearly increase in one direction of space. The direction of maximum increase is called the preferred direction, and it is equivalent to the vector $\vec{W}_{a}$ in Eq. (11). This stands in contrast to the typical visual receptive field found in early visual areas. In V1, for example, receptive fields are typically Gaussian with a half-width of about $0.5^{\circ}$ or less.

The second requirement arises because of the additive interaction between the eye position signal and retinal contribution to the overall activity in Eq. (11).

The third requirement is a consequence of the fact that $\vec{R}$ and $\vec{E}$ in Eq. (11) are multiplied by the same vector $\vec{W}_{a}$ that defines the preferred direction.

In the next section, these three requirements are compared to available neurophysiological recordings of parietal neurons. Neurons are intrinsically nonlinear and it would be unreasonable to expect them to have perfectly linear responses. Furthermore, as we have emphasized before, Eq. (1) is only an approximation (Westheimer, 1957). It is close to the right function for angles less than $40^{\circ}$ - the range of angle typically used in experimentsbut the differences are sufficient to introduce slight nonlinearities in the tuning to $\vec{R}$ and $\vec{E}$, even if the cell is responding linearly to $\vec{A}$.

The key question is whether the nonlinearities are large and functionally significant for the cortex or whether they are small irrelevant deviations from linear responses.

\section{Match Between the Vectorial Representation and Parietal Cortex}

The receptive fields of neurons for eye position are formally equivalent to their gain fields, $80 \%$ of which are linear or contain a linear component in the parietal cortex (Andersen \& Zipser, 1988; Andersen et al., 1990b). This would therefore appear to be consistent with the first prediction of the vectorial hypothesis. However, $20 \%$ are nonlinear, and, as discussed above, the other $80 \%$ may be better described by sigmoids as some of these show clear sign of saturation.

Visual receptive fields in the parietal cortex are not even approximately planar, as shown in Figure 6. They are typically bell-shaped, sometimes with multiple peaks (Andersen et al., 1990a). Only a small fraction of parietal neurons have extremely large receptive fields that cover almost the entire visual field, as predicted for a vectorial code. On average the receptive field diameters are about $44^{\circ}$ in diameter, which is large when compared to earlier visual areas, but still well under the full extent of the visual field $\left(180^{\circ}\right.$ ) (Andersen et al., 1990a).

The second requirement concerns the interaction between the retinal and eye position selectivities. As reported by Andersen et al. (1985) eye position has a multiplicative effect on the visual response of parietal cells, a nonlinear interaction that is incompatible with a vectorial code.

The third prediction made by the vectorial hypothesis regards the visual and eye position preferences of single cells. The only two cells for which both the visual receptive field and the gain field have been published have opposite preferred directions for retinal and eye position (see Figs. 1 and 6 in Andersen \& Zipser, 1988). Clearly more data are needed on the correlation between the preferred eye and retinal position.

In conclusion, the experimental data do not appear to be fully consistent with the predictions of the vectorial code. The visual receptive fields, in particular, are strongly nonlinear. It is still possible, however, that these nonlinearities are averaged out in subsequent stages of processing in the cortex so that the net result is a linear mapping. Most sensorimotor mappings are nonlinear, but, as we argue in the Discussion, there may be particular tasks for which a linear mapping is required.

\section{DISCUSSION}

The fundamental assumption underlying this paper is that spatial representations can be best understood from the perspective of sensorimotor transformation. We propose that the role of spatial representations is to code the sensory inputs and posture signals in a format that simplifies subsequent computation, particularly in the generation of motor commands. This can be achieved by using basis function neurons that reduce the nonlinear transformations involved in sensorimotor coordination to linear mappings.

Available neurophysiological data are consistent with this hypothesis. A neuron with a restricted visual receptive field modulated as a monotonic function of eye position can be modeled by a product of a Gaussian and a sigmoid. Since functions defined as the products of Gaussians and sigmoids form basis functions, this representation can be used to approximate any nonlinear functions of the input variables.

There are two major advantages in reducing the complexity of sensorimotor transformations with basis functions. First, it simplifies learning since the first layer of weights is fixed and only a linear mapping from the hidden layer to the output layer needs to be learned. A simple learning rule, such as the Widrow-Hoff rule used here, or even hebbian mechanisms (Salinas \& Abbott, 1995) could suffice. Second, since the nonlinearities are computed at the level of the basis function units inde- 
pendently of the eventual output, the resulting representation is versatile, in that it contains multiple frames of reference and can be used to control several behaviors simultaneously.

In contrast, previous attempts to characterize spatial representations have emphasized linear encoding schemes, such as the vectorial code (Mazzoni \& Andersen, 1995; Touretzky, Redish, \& Wan, 1993), in which the position of the object is encoded in one particular frame of reference. We have shown that this linear representation is not fully consistent with experimental data from the parietal cortex and is not suitable for nonlinear function approximation. Linear representations are, however, computationally interesting for other operations, such as vector rotation. Regions of the brain more specialized for navigation, such as the hippocampus, may use such a scheme (Touretzky, Redish, \& Wan, 1993).

\section{Comparison with the Zipser and Andersen Network Model}

Zipser and Andersen were the first to provide a network model of the parietal cortex (Zipser \& Andersen, 1988). Their feedforward network was trained with back-propagation to compute the position of an object in head-centered coordinates. The inputs were similar to those in Figure 4 , but the output was trained to represent the head-centered position of the stimulus with either a vectorial code or a map output - the latter case is illustrated in Figure 4. This model made an important contribution toward understanding how neurons in the parietal cortex are used to control behavior, but the nature of the representations found in the hidden layer remained elusive. The analysis presented here provides a conceptual framework for interpreting the hidden representations in the Zipser and Andersen network. This framework can be used to understand why parietal lesions lead to a neurological deficit such as hemineglect (Pouget \& Sejnowski, 1996b; Pouget \& Sejnowski, 1996a).

Previous approaches have focused on networks with a vectorial output (Goodman \& Andersen, 1990; Mazzoni \& Andersen, 1995). In this special case, the overall transformation performed by the network is linear and the hidden layer uses a vectorial code for the head-centered locations of the object (Goodman \& Andersen, 1990). We extend this analysis to the map output, a case that may be particularly relevant for parietal cells given the recent finding of neurons with head-centered receptive fields in the premotor cortex (Fogassi et al., 1992; Graziano, Yap, \& Gross, 1994) and the nonlinear nature of sensorimotor transformations in general. Our approach highlights those aspects of the responses of single parietal neurons that are computationally critical for sensorimotor transformations, such as the nonlinearities found in the retinal and eye position selectivities (particularly in the eye position gain fields) and the nonlinear interactions between them.
An important difference, however, between basis functions and the Zipser and Andersen network is that the hidden unit representation produced by back-propagation is specific for the training that was used to create the network, whereas the basis function representation is independent of the eventual output. The price paid for this versatility is the potentially large number of units that may be needed, since many more units are required for a basis function network than for a specialized backpropagation network.

\section{Modularity in Sensorimotor Coordination}

It is generally believed that sensorimotor coordination involves parallel modules, each dedicated to a particular transformation, such as moving the eye toward a visual target, with each of them embodying its own set of coordinates (see Stein, 1992, for a review). Neurophysiological data from the parietal cortex suggest basis function representations in which several frames of reference are encoded simultaneously by the same neuronal pool. Theoretically, it is possible to collapse all the intermediate steps shown in Figure 1 into a single representation using basis functions spanning all possible combinations of sensory and posture signals. This would provide a representation that could perform any transformation, such as from visual to joint coordinates, in one step and that could be used for all behaviors. In a sense, basis functions implicitly contain all frames of reference at once. In an ideal basis function representation, there would be no need to have parallel modules for each transformation.

A single basis function representation may, however, demand too many neurons since the number of localized basis functions needed to evenly cover an input space increases exponentially with the number of dimensions. Thus, as more signals need to be combined, such as retinal position, eye position, head position, auditory, vestibular, and somatosensory inputs, the number of neurons required eventually exceeds those available, a problem called the "curse of dimensionality."

How can the number of neurons in a basis function representation be minimized? If the repertoire of transformations and the number of output functions is limited, then there is no need to cover the input space with basis functions evenly and the parietal cortex can selectively span the input space to achieve greater efficiency (Moody \& Darken, 1989; Sanger, 1991). There is evidence that some variables are not represented independently in parietal cortex. For example, the response of a neuron in parietal cortex to head position may not be independent of its response to eye position, but these are often correlated along a particular direction in space that is specific to each neuron. This implies that the transformations computed downstream from the parietal cortex do not need to distinguish explicitly between head and eye position. 
Even when the dimension of the input space can be reduced, a single representation might be cumbersome. A compromise between extreme modularity (one module for each frame of reference) and multipurpose basis functions is possible. Sensorimotor transformations might be decomposed in several steps, whether sequentially or in parallel, but each of these intermediate transformations may involve basis function modules, instead of a single reference frame. Each of these modules would contain two or three frames of reference and, as such, could be involved in several types of behavior, thereby greatly facilitating crosstalk and coordination. Our model, then, can be applied to each module, or cortical area, individually.

\section{Predictions for Hemineglect}

The ability of basis functions to support multiple reference frames can be tested. One strong prediction is that hemineglect resulting from lesions in the parietal cortex should not be confined to a particular frame of reference. Recent studies of parietal patients are consistent with this conclusion (Ladavas, 1987; Calvanio, Petrone, \& Levine, 1987; Driver \& Halligan, 1991; Behrmann \& Moscovitch, 1994). The experiments of Ladavas (1987) and Calvanio et al. (1987), for example, show that the deficit is both retinotopic and environmental. Our explanation reconciles these observations with the properties of single cells in the parietal cortex.

We tested this explanation of hemineglect by lesioning our basis function model and comparing the pattern of breakdown with deficits reported in patients with a variety of parietal lesions. Preliminary results from our simulations indicate strong similarities between the behavior of our model and observations on patients with hemineglect (Pouget \& Sejnowski, 1996b; Pouget \& Sejnowski, 1996a).

\section{Predictions for Visuo-Motor and Perceptual Adaptation Experiments}

Human subjects wearing visual prisms can learn in fewer than 20 trials to reach accurately for a visual target. In a recent study, Ghahramani, Wolpert, and Jordan (1995) investigated the pattern of generalization after prism adaptation to one spatial location. Their results suggested that adaptation takes place in Cartesian space rather than joint coordinates. The frame of reference of this Cartesian space, however, could not be determined from their experiments.

It is conceivable that learning takes place in bodycentered coordinates. Alternatively, if basis function neurons are involved in these experiments, the adaptation might occur in the space defined by the basis functions, a space whose axes are retinal location and eye position. This would predict that manipulation of gaze angle should affect the pattern of generalization in the Gharahmani et al. (1995) experiment, even when the position of the target is kept fixed in body-centered coordinates.

The basis function framework also predicts that eye position could similarly influence perceptual adaptation experiments. A motion after-effect study by Mayhew (1973) supports this possibility. Subjects were asked to alternate fixation between a clockwise rotating spiral located on their right and a counterclockwise rotating spiral located on their left. After a few minutes, the spiral motion was stopped and subjects reported a counterclockwise motion after-effect for the right spiral and a clockwise motion after-effect for the left spiral. Other perceptual after-effects might reveal a similar dependency on eye position.

\section{Beyond Parietal Cortex}

Although the focus of this paper was on the response properties of parietal neurons, our approach can be generalized to any cortical area where gain modulation of a sensory response by a posture signal has been reported. Our basis function framework predicts that gain modulation should be found in cortical areas located at the interface between the sensory and motor systems. Gain modulation has already been found in several cortical areas, in particular the supplementary eye field (Schall, 1991), the ventral premotor cortex (Boussaoud, Barth, \& Wise, 1993), and the parietal area 7b (Field \& Olson, 1994).

Modulation of visual responses by eye position has also been observed in the striate cortex (Trotter et al., 1992; Weyand \& Malpeli, 1993), area V3a (Galletti \& Battaglini, 1989), parietal area DP (Andersen et al., 1990a), and even in the lateral geniculate nucleus (Lal \& Friedlander, 1989), areas in which the receptive fields form retinotopic maps. Since Gaussian functions form a basis set regardless of their width (Baldi, 1991), our hypothesis can be readily extended to early visual areas where cells have receptive fields with small widths. As we have suggested in a previous study (Pouget, Fisher, \& Sejnowski, 1993), the smaller size of the visual receptive fields in V3a could provide a spatial representation of object subparts, whereas area $7 \mathrm{a}$ might be more concerned with whole objects.

Therefore, the basis function hypothesis might be applicable to spatial representations outside of the parietal cortex, from the primary visual cortex to the premotor cortex (Pouget, Fisher, \& Sejnowski, 1993; Pouget \& Sejnowski, 1994).

\section{Appendix}

Part 1: The Product of Two Basis Sets Forms a Basis Set

We first demonstrate that if the sets $\left\{G_{i}(x)\right\}_{i=0}^{\infty}$ and $\left\{S_{j}(y)\right\}_{j=0}^{\infty}$ form complete basis sets, then the set $\left\{G_{i}(x) S_{j}(y)\right\}_{i=0, j=0}^{\infty}$ is complete. We use the following (Keener, 1988, p. 70, theorem 2.2): 
Theorem 1 A set $\left\{B_{k}(x)\right\}_{k=0}^{\infty}$ is complete iff, if $\left\langle f, B_{k}\right\rangle$ $=0$ for all $k$, then $f=0$.

Where

$$
\left\langle f, B_{k}\right\rangle=\int_{-\infty}^{\infty} \int_{-\infty}^{\infty} f(x, y) B_{k}(x, y) d x d y
$$

Assume that a function $f(x, y)$ satisfies

$$
\left\langle f, G_{i} S_{j}\right\rangle
$$

for all $i$ and $j$.Then

$$
\begin{aligned}
& \int_{-\infty}^{\infty} \int_{-\infty}^{\infty} f(x, y) G_{i}(x) S_{j}(y) d x d y=0 \\
& \int_{-\infty}^{\infty}\left(\int_{-\infty}^{\infty} f(x, y) G_{i}(x) d x\right) S_{j}(y) d y=0
\end{aligned}
$$

Let

$$
g(y)=\int_{-\infty}^{\infty} f(x, y) G_{i}(x) d x
$$

Replacing $g(y)$ in Eq. (15) leads to:

$$
\begin{gathered}
\int_{-\infty}^{\infty} g(y) S_{j}(y) d y=0 . \\
\left\langle g, S_{j}\right\rangle=0 .
\end{gathered}
$$

Since $\left\{S_{j}\right\}_{j=0}^{\infty}$ is complete, $g=0$ and

$$
\begin{aligned}
\int_{-\infty}^{\infty} f(x, y) G_{i}(x) d x & =0, \\
\left\langle f, G_{i}\right\rangle & =0 .
\end{aligned}
$$

Since $\left\{G_{i}(x)\right\}_{i=0}^{\infty}$ is also complete, then $f=0$. Therefore, we have shown that if $\left\langle f, G_{i} S_{j}\right\rangle=0$ then $f=0$. It follows that $\left\{G_{i}(x) S_{j}(y)\right\}_{i=0, j=0}^{\infty}$ forms a basis set.

\section{Part 2: Necessary Conditions for Basis Functions}

We show that any set that does not satisfy the two conditions in the section on "Response Properties Required by Basis Function Representations" cannot form a basis set. The first condition states that the selectivities to $\vec{R}$ and $\vec{E}$ should interact nonlinearly.

To demonstrate that this is a necessary condition, we need to show that no set of the form $\left\{a_{i j} D_{i}(x)+\right.$ $\left.b_{i j} H_{j}(y)\right\}_{i=0, j=0}^{\infty}$ is complete, where $\left\{a_{i j}, b_{i j}\right\}_{i=0, j=0}^{\infty}$ is a set of fixed coefficients.

Consider a function $f$ such that $\left\langle f, a_{i j} D_{i}+b_{i j} H_{j}\right\rangle=0$ :

$$
\begin{aligned}
& \int_{-\infty}^{\infty} \int_{-\infty}^{\infty} f(x, y)\left(a_{i j} D_{i}(x)+b_{i j} H_{j}(y)\right) d x d y=0, \\
& \int_{-\infty}^{\infty} \int_{-\infty}^{\infty} f(x, y)\left(a_{i j} D_{i}(x)\right) d x d y \\
&+\int_{-\infty}^{\infty} \int_{-\infty}^{\infty} f(x, y)\left(b_{i j} H_{j}(y)\right) d x d y=0,
\end{aligned}
$$

$$
\begin{aligned}
\int_{-\infty}^{\infty} a_{i j} D_{i}(x)\left(\int_{-\infty}^{\infty} f(x, y) d y\right) d x \\
+\int_{-\infty}^{\infty} b_{i j} H_{j}(y)\left(\int_{-\infty}^{\infty} f(x, y) d x\right) d y=0 .
\end{aligned}
$$

This expression is true for any $f$ such that:

$$
\left\{\begin{array}{l}
\int_{-\infty}^{\infty} f(x, y) d y=0 \\
\int_{-\infty}^{\infty} f(x, y) d x=0
\end{array}\right.
$$

Any odd functions in $x$ and $y$, i.e., functions such that $f(x, y)=-f(-x,-y)$, such as $\exp \left(-(x+y)^{2}\right) \sin (x+y)$, satisfy these two equalities. Therefore, there exists a function $f$, different from the null function, such that $\left\langle f, E_{i}+H_{j}\right\rangle=0$, from which we can conclude that the set $\left\{E_{i}+H_{j}\right\}_{i=0, j=0}^{\infty}$ is not complete.

A set of functions composed of two sets of functions, one from functions of $x$ only and the other from functions of $y$ only, $\left\{\left\{D_{i}(x)\right\}_{i=0}^{\infty}, \quad\left\{H_{i}(y)\right\}_{i=0}^{\infty}\right\}$ is a subcase of the previous case.

Consequently $\left\{\left\{D_{i}(x)\right\}_{i=0}^{\infty},\left\{H_{i}(y)\right\}_{i=0}^{\infty}\right\}$ cannot form a basis set. This implies that a representation in which units represent $\vec{R}$ and $\vec{E}$ with distinct neuronal populations does not contain a basis set.

We now turn to the second condition, in which the visual receptive fields as well as the gain fields should be nonlinear functions of $\vec{R}$ and $\vec{E}$.

To demonstrate that this is a necessary condition, we need to show that if response functions of parietal neurons are linear in $\vec{R}$ and $\vec{E}$, they cannot form a basis set. If the tunings are linear, the response function can be only of the form:

$$
f(\vec{R}, \vec{E})=a+b \overrightarrow{W_{1}^{R}} \vec{R}+c \vec{W}_{1}^{R} \vec{E}+d\left(\vec{W}_{2}^{R} \vec{R}\right)\left(\overrightarrow{W_{2}^{E}} \vec{E}\right)
$$

Consequently, a linear combination of such functions can be used to approximate polynomial of second degree only (e.g., $f(x)=a+b x+c x^{2}$, in 1-D), which is a restricted set of nonlinear functions.

Therefore, a set of functions that do not meet the two conditions we have proposed cannot form a basis set, from which we can conclude that these two conditions are necessary.

\section{Acknowledgments}

This research was supported in part by a fellowship from the McDonnell-Pew Center for Cognitive Neuroscience in San Diego (A.P.) and the Office of Naval Research (T.J.S.). We thank Peter Dayan, Daphne Bavelier, Richard Zemel and Laurenz Wiskott for their valuable comments and suggestions. We are also grateful to Richard Andersen for providing the data used for Figure 9 and for helpful comments on this manuscript.

Reprint requests should be sent to Alexandre Pouget, New Research Bldg. EP04, 3970 Reservoir Road, Washington, D.C. 20007. Email: alex@salk.edu. 


\section{REFERENCES}

Andersen, R. (1989). Visual and eye movement functions of the posterior parietal cortex. Annual Review in Neuroscience, 12, 377-403.

Andersen, R. (1995). Encoding of intention and spatial location in the posterior parietal cortex. Cerebral Cortex, 5(5), 457-469.

Andersen, R., Asanuma, C., Essick, G., \& Siegel, R. (1990a). Corticocortical connections of anatomically and physiologically defined subdivisions within the inferior parietal lobe. Journal of Comparative Neurology, 296(1), 65-113.

Andersen, R., Bracewell, R., Barash, S., Gnadt, J., \& Fogassi, L. (1990b). Eye position effect on visual memory and saccade-related activity in areas LIP and $7 \mathrm{a}$ of macaque. Journal of Neuroscience, 10, 1176-1196.

Andersen, R., Essick, G., \& Siegel, R. (1985). Encoding of spatial location by posterior parietal neurons. Science, 230, 456-458.

Andersen, R., \& Zipser, D. (1988). The role of the posterior parietal cortex in coordinate transformations for visualmotor integration. Canadian Journal of Physiology and Pharmacology, 66, 488-501.

Baldi, P. (1991). Computing with arrays of bell-shaped and sigmoid functions. In R. Lippman, J. Moody, \& D. Touretzky (Eds.), Advances in neural information processing systems (Vol. 3). San Mateo, CA: Morgan-Kaufmann.

Behrmann, M., \& Moscovitch, M. (1994). Object-centered neglect in patients with unilateral neglect: Effects of leftright coordinates of objects. Journal of Cognitive Neuroscience, 6(2), 151-155.

Blatt, G., Andersen, R., \& Stoner, G. (1990). Visual receptive field organization and corticocortical connections of the lateral intraparietal area (area LIP) in the macaque. Journal of Comparative Neurology, 299(4), 421-445.

Boussaoud, D., Barth, T., \& Wise, S. (1993). Effects of gaze on apparent visual responses of frontal cortex neurons. $E x$ perimental Brain Research, 93(3), 423-434.

Burnod, Y., Grandguillaume, P., Otto, I., Ferraina, S., Johnson, P., \& Caminiti, R. (1992). Visuomotor transformations underlying arm movements toward visual targets: A neural network model of cerebral cortical operations. Journal of Neuroscience, 12(4), 1435-1453.

Calvanio, R., Petrone, P., \& Levine, D. (1987). Left visual spatial neglect is both environment-centered and bodycentered. Neurology, 37, 1179-1181.

Casdagli, M. (1989). Nonlinear prediction of chaotic time series. Pbysica D, 35, 335-356.

Colby, C., \& Duhamel, J. (1993). Ventral intraparietal area of the macaque: Anatomic location and visual response properties. Journal of Neuropbysiology, 69(3), 902-914.

Craig, J. (1955). Introduction to robotics. Redwood City, CA: Addison Wesley.

Driver, J., \& Halligan, P. (1991). Can visual neglect operate in object-centered coordinates? An affirmative single case study. Cognitive Neuropsychology, 8(6), 475-496.

Droulez, J., \& Berthoz, A. (1991). A neural model of sensoritopic maps with predictive short-term memory properties. Proceedings of the National Academy of Science, 88, 9653-9657.

Felleman, D., \& Van Essen, D. (1991). Distributed hierarchical processing in the primate cerebral cortex. Cerebral Cortex, $1,1-47$.

Field, P., \& Olson, C. (1994). Spatial analysis of somatosensory and visual stimuli by single neurons in macaque area $7 \mathrm{~b}$. Society for Neuroscience Abstracts, 20.

Fogassi, L., Gallese, V., di Pellegrino, G., Fadiga, L., Gentilucci, M., Luppino, G., Matelli, M., Pedotti, A., \& Rizzolatti, G.
(1992). Space coding by premotor cortex. Experimental Brain Research, 89(3), 686-690.

Galletti, C., \& Battaglini, P. (1989). Gaze-dependent visual neurons in area V3a of monkey prestriate cortex. Journal of Neuroscience, 9, 1112-1125.

Galletti, C., Battaglini, P., \& Fattori, P. (1993). Parietal neurons encoding spatial locations in craniotopic coordinates. $E x$ perimental Brain Research, 96, 221-229.

Georgopoulos, A., Lurito, J., Petrides, M., Schwartz, A., \& Massey, J. (1989). Mental rotation of the neuronal population vector. Science, 243, 234-236.

Ghahramani, Z., Wolpert, D., \& Jordan, M. (1995). Computational structure of coordinate transformations: A generalization study. In G. Tesauro, D. Touretzky, and T. Leen (Eds.), Advances in neural information processing systems (Vol. 7). Cambridge, MA: Morgan-Kaufmann, MIT Press.

Girosi, F, Jones, M., \& Poggio, T. (1995). Regularization theory and neural network architectures. Neural Computation, 7, 219-269.

Goodman, S., \& Andersen, R. (1990). Algorithm programmed by a neural model for coordinate transformation. In International Joint Conference on Neural Networks, San Diego.

Graziano, M., \& Gross, C. (1993). A bimodal map of space-somatosensory receptive fields in the macaque putamen with corresponding visual receptive fields. Experimental Brain Research, 97(1), 96-109.

Graziano, M., Yap, G., \& Gross, C. (1994). Coding of visual space by premotor neurons. Science, 266, 1054-1057.

Groh, J., \& Sparks, D. (1992). Two models for transforming auditory signals from head-centered to eye-centered coordinates. Biological Cybernetics, 67(4), 291-302.

Groh, J., \& Sparks, D. (1996). Saccades to somatosensory targets 3 Eye-position-dependent somatosensory activity in the primate superior colliculus. Journal of Neurophysiology, 75(1), 439-453.

Heilman, K., Watson, R., \& Valenstein, E. (1985). Neglect and related disorders. In K. Heilman, \& E. Valenstein (Eds.), Clinical neuropsycbology (pp. 243-294). New York: Oxford University Press.

Hornik, K., Stinchcombe, M., \& White, H. (1989). Multilayer feedforward networks are universal approximators. Neural Networks, 2, 359-366.

Jay, M., \& Sparks, D. (1987). Sensorimotor integration in the primate superior colliculus: 1. Motor convergence. Journal of Neurophysiology, 57, 22-34.

Keener, J. (1988). Principles of applied matbematics. Transformation and approximation. Redwood City, CA: Addison Wesley.

Knudsen, E., du Lac, S., \& Esterly, S. (1987). Computational maps in the brain. Annual Review of Neuroscience, 10 , 41-65.

Ladavas, E. (1987). Is the hemispatial deficit produced by right parietal lobe damage associated with retinal or gravitational coordinates? Brain, 110, 167-180.

Lal, R., \& Friedlander, M. (1989). Gating of retinal transmission by afferent eye position and movement signals. Sctence, 243, 93-96.

Lee, C., Rohrer, W., \& Sparks, D. (1988). Population coding of saccadic eye movements by neurons in the superior colliculus. Nature, 332 (6162), 357-360.

Logothetis, N., \& Pauls, J. (1995). Psychophysical and physiological evidence for viewer-centered object representations in the primate. Cerebral Cortex, 3, 270-288.

Mayhew, J. (1973). After-effects of movement contingent on direction of gaze. Vision Research, 13, 877-880.

Mazzoni, P., \& Andersen, R. (1995). Gaze coding in the posterior parietal cortex. In M. Arbib (Ed.), The handbook of 
brain theory and neural networks (pp. 423-426). Cambridge, MA: MIT Press.

Moody, J., \& Darken, C. (1989). Fast learning in networks of locally tuned processing units. Neural Computation, 1, 281-294.

Poggio, T. (1990). A theory of how the brain might work. Cold Spring Harbor Symposium on Quantitative Biology, 55, 899-910.

Poggio, T., \& Edelman, S. (1990). A network that learns to recognize three-dimensional objects. Nature, 343(6255), 263266.

Poggio, T., \& Girosi, F. (1990). Regularization algorithms for learning that are equivalent to multilayer networks. Science, 247, 978-982.

Pouget, A., Fisher, S., \& Sejnowski, T. (1993). Egocentric spatial representation in early vision. Journal of Cognitive Neuroscience, 5, 150-161.

Pouget, A., Montague, P., Dayan, P., \& Sejnowski, T. (1993). A developmental model of map registration in the superior colliculus using predictive hebbian learning. Society for Neuroscience Abstracts, 19.

Pouget, A., \& Sejnowski, 'T. (1994). A neural model for the cortical representation of egocentric distance. Cerebral Cortex, 4, 314-329.

Pouget, A., \& Sejnowski, 'T. (1995). Spatial representations in the parietal cortex may use basis functions. In G. Tesauro, D. Touretzky, \& T. Leen (Eds.), Advances in neural information processing systems (Vol. 7). Cambridge, MA: MIT Press.

Pouget, A., \& Sejnowski, T. (1996a). Lesion in a basis function model of spatial representations: Comparison with hemineglect. In P. Thier, \& H. Karnath (Eds.), Parietal lobe contribution in orientation in $3 D$ space.

Pouget, A., \& Sejnowski, T. (1996b). A model of spatial representations in parietal cortex explains hemineglect. In D. Touretzky, M. Mozer, \& M. Hasselman (Eds.), Advances in neural information processing systems (Vol. 8). Cambridge, MA: MIT Press.

Salinas, E., \& Abbott, L. (1995). Transfer of coded information from sensory to motor networks. Journal of Neuroscience, 15 (10), 6461-6474.
Sanger, T. (1991). Basis-function trees as a generalization of local variable selection methods for function approximation. In R. Lippmann, J. Moody, \& D. Touretzky (Eds.), Advances in neural information processing systems (Vol. 3, pp. 700-706). San Mateo, CA: Morgan Kaufmann.

Sanger, T. (1994). Theoretical consideration for the analysis of population coding in motor cortex. Neural Computation, 6, 29-37.

Schall, J. (1991). Neuronal activity related to visually guided saccadic eye movements in the supplementary motor area of thesus monkeys. Journal of Neurophysiology, 66(2), 530-558.

Soechting, J., \& Flanders, M. (1992). Moving in three-dimensional space: Frames of reference, vectors and coordinate systems. Annual Review in Neuroscience, 15, 167-191.

Sparks, D. (1991). Sensorimotor integration in the primate superior colliculus. Seminars in the Neurosciences, 3, 39-50.

Squatrito, S., \& Maioli, M. (1996). Gaze field properties of eye position neurones in areas MST and $7 \mathrm{a}$ of macaque monkey. Visual Neuroscience, 13, 385-398.

Stein, J. (1992). The representation of egocentric space in the posterior parietal cortex. Bebavioral and Brain Sciences, 15(4), 691-700.

Touretzky, D., Redish, A., \& Wan, H. (1993). Neural representation of space using sinusoidal arrays. Neural Computation, 5, 869-884.

Trotter, Y., Celebrini, S., Stricanne, B., Thorpe, S., \& Imbert, M. (1992). Modulation of neural stereoscopic processing in primate area V1 by the viewing distance. Science, 257, 1279-1281.

Westheimer, G. (1957). Kinematic of the eye. Journal of the Optical Society of America, 47(1), 967-974.

Weyand, T., \& Malpeli, J. (1993). Responses of neurons in primary visual cortex are modulated by cye position. Journal of Neurophysiology, 69(6), 2258-2260.

Widrow, B., \& Hoff, M. (1960). Adaptative switching circuits. IRE WESCON Convention Records, 4, 96-104.

Zipser, D., \& Andersen, R. (1988). A back-propagation programmed network that stimulates response properties of a subset of posterior parietal neurons. Nature, 331, 679684. 